\title{
Interventions might offer a pregnant pause in addiction
}

Doctors have known for decades that cigarettes and alcohol harm developing babies. Now, because many pregnant women find it difficult to beat these addictions, research has begun looking for ways to minimize their damage to the fetus.

For instance, although governments recommend that pregnant women stop smoking, they don't usually recommend nicotine replacement therapy (NRT), owing to a lack of research on its safety and efficacy in pregnant women. Meanwhile, despite decades of warnings that smoking causes problems such as stillbirths and miscarriage, many pregnant smokers don't quit- $17 \%$ of women smoke throughout pregnancy in Britain, for instance.

Preliminary studies have offered both good and bad news about nicotine replacement therapy in pregnant smokers. In 2000, a Danish group published the only large placebocontrolled trial on NRT to date in 250 women (Obstet. Gynecol. 96, 967-971; 2000). Although NRT didn't help women stop smoking, the trial found that NRT users gave birth to heavier infants than those who did not receive the nicotine therapy.

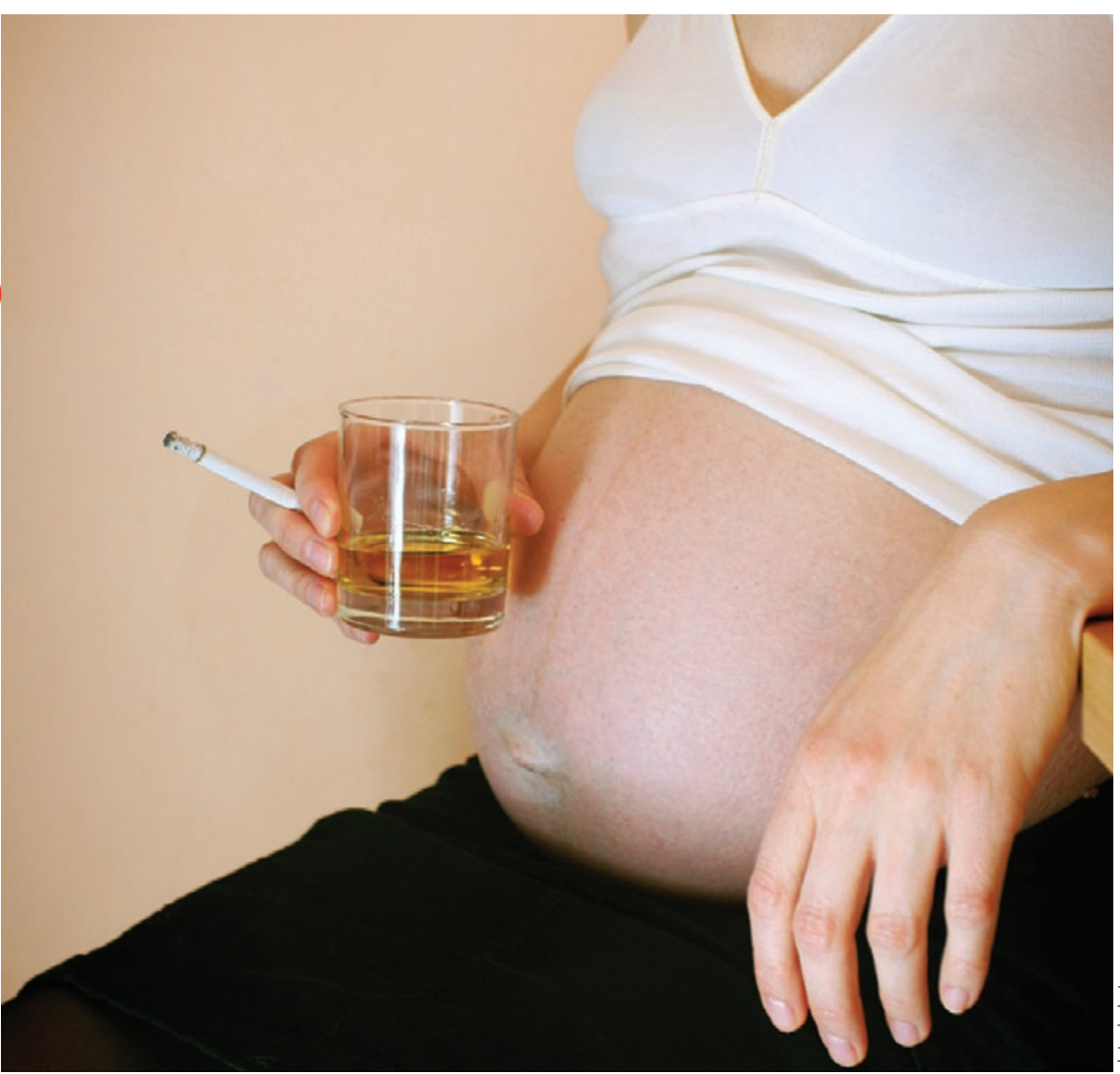

Smoking for two: Several recent studies have looked at whether nicotine replacement might help

Clouding the picture, a 2006 observational study found an elevated risk of birth defects due to NRT. But other studies have contradicted those results (Obstet. Gynecol. 107, 51-57; 2006). And a study published in August found that pregnant women receiving NRT did not have higher rates of stillbirth than smokers who did not use NRT (BJOG 115, 1405-1410; 2008). The study also found that women who used NRT had lower rates of stillbirth than those who smoked and did not use NRT, but the trend didn't reach statistical significance.

According to Tim Coleman of the University of Nottingham, the evidence so far suggests that NRT is safer than smoking for the fetus. But he adds that the picture remains incomplete. "It may well be safe to use nicotine to keep you away from cigarettes, because once you light a cigarette you expose yourself to all other sorts of horrible things," says Coleman, who is now recruiting 1,050 pregnant women for a placebo-controlled NRT trial sponsored by the UK's National Institute for Health Research Health Technology Assessment Programme. Meanwhile, a study published in September hinted that exercise might help women quit smoking during pregnancy $(B M C$

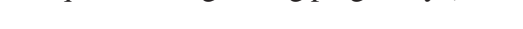

